\title{
ASSOCIAÇÃO ENTRE A PRESENÇA DE TRANSTORNOS DE ANSIEDADE COM O SEXO EM ADOLESCENTES ESCOLARES
}

\author{
ASSOCIATION BETWEEN THE PRESENCE OF ANXIETY \\ DISORDERS WITH SEX IN SCHOOL TEENAGERS
}

\author{
Lucenildo Laerty da Silva Sales ${ }^{1}$ \\ José Ewayr Mariano de Araújo ${ }^{2}$ \\ Elayne Maria Cordeiro Costa ${ }^{3}$ \\ Carla Campos Muniz Medeiros ${ }^{4}$ \\ Danielle Franklin de Carvalho ${ }^{5}$
}

RESUMO: OBJETIVO: Identificar a presença de Transtornos de Ansiedade (TA) associado com o sexo em adolescentes escolares de escolas públicas do município de Campina Grande - Paraíba. METODOLOGIA: Estudo transversal realizado com 131 adolescentes, entre 15 e 19 anos, matriculados em duas escolas públicas de ensino médio integral da zona urbana do município de Campina Grande - Paraíba. Para a avaliação da presença de TA, utilizou-se o Questionário para Triagem de Ansiedade Infantil (QTAI-C). Foi aprovado pelo comitê de ética da Universidade Estadual da Paraíba sob o CAAE n 0077.0.133.000-12. Para processamento dos dados, utilizou-se o Statistical Package for the Social Sciences (SPPS) versão 21.0. Realizou-se análise descritiva das variáveis estudadas e o teste qui-quadrado para avaliação da associação do sexo com os TA, considerando intervalo de confiança de 95\%. RESULTADOS E DISCUSSÕES: Da amostra selecionada, $58 \%$ eram do sexo feminino e $42 \%$ eram do sexo masculino. Constatou-se que $32,1 \%$ dos alunos participantes, possuíam algum dos Transtornos de Ansiedade (TAs), sendo a maior prevalência nos estudantes do sexo feminino (40,8\%). CONCLUSÃO: Este resultado

\footnotetext{
1 Enfermeiro. Especialista em Gestão e Auditoria em Serviços de Saúde. Mestrando em Saúde Pública - PPGSP/UEPB.

2 Farmacêutico. Especialista em Farmacologia clínica e atenção farmacêutica. Mestrando em Saúde Pública -

PPGSP/UEPB

3 Enfermeira. Especialista em Urgência, Emergência e UTI. Mestre em Saúde Pública PPGSP/UEPB.

${ }^{4}$ Médica. Professora do Programa de Pós-Graduação em Saúde Pública. Doutora em Saúde da Criança e Adolescente.

${ }^{5}$ Farmacêutica. Professora do Programa de Pós-Graduação em Saúde Pública. Doutora em Saúde da Criança e Adolescente.
} 
reflete e é somado com diversos outros estudos que demonstram esta associação com o gênero. Isso é apontando por diversos autores, considerando as alterações fisiológicas e hormonais desta fase como preditor do comportamento ansioso. Portanto, é necessária a investigação mais profunda desta associação visando traçar estratégias de cuidado no contexto social, além de esclarecer as reais causas desta associação.

Palavras chave: Adolescente; Ansiedade; Transtornos Fóbicos.

ABSTRACT: OBJECTIVE: To identify the presence of Anxiety Disorders (ED) associated with sex in school adolescents from public schools in the city of Campina Grande - Paraiba. METHODOLOGY: Cross-sectional study carried out with 131 adolescents, between 15 and 19 years old, enrolled in two public high schools in the urban area of the city of Campina Grande - Paraíba. To assess the presence of ED, the Child Anxiety Screening Questionnaire (QTAI-C) was used. It was approved by the ethics committee of the Universidade Estadual da Paraíba under CAAE $n^{\circ}$ 0077.0.133.000-12. For data processing, the Statistical Package for the Social Sciences (SPPS) version 21.0 was used. A descriptive analysis of the studied variables and the chi-square test were performed to assess the association of sex with ED, considering a 95\% confidence interval. RESULTS AND DISCUSSIONS: Of the selected sample, $58 \%$ were female and $42 \%$ were male. It was found that $32.1 \%$ of the participating students had some of the Anxiety Disorders (EDs), with the highest prevalence among female students (40.8\%). CONCLUSION: This result reflects and is added to several other studies that demonstrate this association with gender. This is pointed out by several authors, considering the physiological and hormonal changes of this phase as a predictor of anxious behavior. Therefore, it is necessary to further investigate this association in order to outline care strategies in the social context, in addition to clarifying the real causes of this association.

Keywords: Adolescent; Anxiety; Phobic Disorders. 


\section{INTRODUÇÃO}

A adolescência é compreendida como um período do desenvolvimento humano no qual intensas mudanças hormonais, físicas, emocionais, cognitivas, sociais e psicológicas são observadas. Uma pesquisa realizada no ano de 2011, traz que cerca de 21 milhões de adolescentes vivem no Brasil, correspondendo a $21 \%$ da população brasileira (MEÁ; BIFFE; FERREIRA, 2016).

No Brasil, considera-se adolescente o indivíduo entre doze e dezoito anos de idade. Nesta fase de desenvolvimento, este grupo está sujeito à constantes dúvidas e desafios diante das mudanças experimentadas, sendo estas, preditoras de alterações psicoafetivas. Sintomas depressivos e de ansiedade são fenômenos recorrentes na adolescência e precisam de atenção para que não causem prejuízos a longo prazo (GROLLI; WAGNER; DALBOSCO, 2017).

Situações como a busca e a formação da identidade, variações de humor com mediação de conflitos e desentendimentos com amigos e familiares, constituem momentos de desafios para o adolescente que precisa lidar de forma coerente com o enfrentamento destes eventos. Isto nem sempre acontece de forma positiva, o que leva o indivíduo adolescente a um período de vulnerabilidade emocional, sendo este, o momento propício à instalação de sintomas depressivos e de ansiedade (GROLLI; WAGNER; DALBOSCO, 2017).

Entende-se por Transtornos de Ansiedade (TAs), episódios que compartilham características de medo e ansiedade excessivos, persistindo além dos períodos apropriados ao nível de desenvolvimento (ALMEIDA et al., 2016). Constitui um dos problemas de saúde pública observados na atual conjuntura, apresentando altas taxas de prevalência e afetando de forma negativa a qualidade de vida dos indivíduos. Estudos mostram que uma criança apresenta, hoje em dia, o mesmo nível de ansiedade observado em pacientes psiquiátricos no ano de 1950 (SILVA, C. B., 2016). 
Os problemas emocionais e de conduta constituem uma carga de doença expressiva $(10 \%$ - 20\%) em crianças e adolescentes, causando prejuízos nas relações familiares, sociais e na vida escolar. Os transtornos psiquiátricos são apontados como uma das causas de anos de vida perdidos por incapacidade e adolescentes e jovens entre 10 e 24 anos (LOPES et al., 2016).

Os TAs prejudicam a vida diária dos indivíduos, com interrupção de atividades rotineiras devido ao medo das crises e sintomas, afetando assim a qualidade de vida, diminuindo o grau de independência e causando rompimentos sociais (COSTA et al., 2019). Além disso, é válido ressaltar que grande parte dos TAs que têm início na infância, necessitam de tratamento específico e adequado objetivando que estes não se prolonguem para a vida adulta (SILVA, C. B., 2016).

Ainda, os TAs ocasionam manifestações somáticas, a exemplo de cefaleia, dispneia, taquicardia, tremores, vertigem, sudorese, parestesias, náuseas e diarreia etc., além de psíquicas, como inquietação interna, insegurança, insônia, irritabilidade, desconforto mental, dificuldade de concentração, etc. (DA SILVA FILHO; DA SILVA, 2013).

Uma investigação realizada no ano de 2016 com escolares do ensino médio, identificou a presença significativa de sintomas depressivos e de ansiedade nestes indivíduos. As meninas apresentaram estes sintomas em maior quantidade em relação aos meninos, bem como uma ideia negativa de apoio dos amigos e familiares em situações da vida diária (GROLLI; WAGNER; DALBOSCO, 2017).

Ainda, Souza (2011) complementa, afirmando que os transtornos de ansiedade estão entre os mais comumente observados na população em geral, estando associados a altas taxas de psicopatologia na adolescência e na vida adulta. (SOUZA, 2011).

A existência de medo e ansiedade, em proporções normais, diminui as "ações irresponsáveis" predispostos pela idade, reduzindo a morbidade em adolescentes. Porém, é comum nos TAs em todas as idades que essas preocupações irracionais acarretem em queixas somáticas que interferem diretamente na qualidade de vida e no bem estar emocional. (DA SILVA FILHO; SILVA, 2017).

Existe um quadro restrito quando se considera a visão multidimensional da ansiedade, buscando diferenças entre o sexo masculino e o feminino. A literatura 
que aponta essa diferença é restrita, porém, seus resultados tem demonstrado que as mulheres apresentam maiores níveis de ansiedade do que os homens (MANOEL, 2017).

Ainda, uma explicação alternativa para as prováveis diferenças entre homens e mulheres refere-se à maneira como os sintomas ansiosos são descritos. As mulheres são mais propensas a relatar seus sintomas do que os homens, principalmente quando nos referimos a sintomas desagradáveis. Em suma, é socialmente aceito que mulheres apontem seus sintomas de ansiedade, enquanto dos homens espera-se menos manifestações das emoções (MANOEL, 2017).

Frequentemente, observa-se que muitos indivíduos, especialmente do sexo feminino, vivem em busca de um "padrão de beleza" imposto pela mídia, sacrificando-se constantemente tentando manter seus corpos magros, esbeltos, de forma frenética, tornando-se escravos de modelos estéticos e padrões sociais (DA SILVA; LANGE, 2017).

Porém, esses padrões modificam-se em grande velocidade, tornando-se cada vez mais difícil segui-los. Isso acarreta no desenvolvimento de transtornos de ansiedade e reforça o fato deles serem mais frequentes no sexo feminino. Caso isso não haja aceitação da autoimagem feminina, o indivíduo terá prejuízo emocional por idealizar uma imagem corporal que contraria a real, uma vez que essa foge aos padrões impostos pela sociedade. (DA SILVA; LANGE, 2017).

Sendo assim, este trabalho busca analisar a prevalência dos transtornos de ansiedade em adolescentes escolares, submetidos às situações reais do ambiente educacional do ensino médio em escolas públicas do município de Campina Grande - PB.

\section{OBJETIVO}

Identificar a prevalência de Transtornos de Ansiedade em adolescentes escolares em adolescentes de ensino médio, matriculados regularmente em escolas públicas do município de Campina Grande - PB. 


\section{METODOLOGIA}

Trata-se de um estudo transversal, realizado com adolescentes matriculados no ensino médio integral de escolas públicas localizadas na zona urbana do município de Campina Grande - Paraíba. Foram escolhidas duas escolas públicas de grande porte do mesmo município, nas quais 131 adolescentes escolares foram selecionados e avaliados.

Adolescentes com idade entre 15 e 19 anos devidamente matriculados e que cursassem as duas primeiras séries do ensino médio integral nas duas escolas públicas foram os critérios de inclusão. Como critérios de exclusão, adotou-se histórico ou em tratamento de algum transtorno de ansiedade diagnosticado e aqueles que estavam cursando o terceiro ano do ensino médio, visto que essa fase exerce influência sobre a saúde mental.

O estudo teve como variável os Transtornos de Ansiedade (TAs), avaliados através da aplicação do Questionário para Triagem de Ansiedade Infantil (QTAI-C), composto por 41 perguntas nas quais, cada uma delas permite 3 opções de resposta seguindo uma classificação para determinação de presença ou ausência de sintomas ansiosos.

Após seleção das escolas, foi realizada visita dos pesquisadores às instituições para eventual explicação dos objetivos do estudo, programação de coleta de dados e agendamento de reunião com pais e alunos para assinatura do Termo de Consentimento Livre e Esclarecido (TCLE) e do Termo de Assentimento Livre e Esclarecido (TALE). Os escolares que aceitaram participar do estudo, responderam os instrumentos da pesquisa com informações socioeconômicas e o questionário para triagem da ansiedade.

Este estudo foi aprovado pelo comitê de ética da Universidade Estadual da Paraíba sob o CAAE n 0077.0.133.000-12 em conformidade com a Resolução ${ }^{\circ}$ 466/12 do Conselho Nacional de Saúde, respeitando os princípios da autonomia, não-maleficência, beneficência, justiça e equidade. 
Após coleta, os dados foram duplamente digitados e validados pelo Validate do Epi Info 6.04. Para processamento das análises estatísticas, utilizou-se o Statistical Package for the Social Sciences (SPPS) versão 21.0. Realizou-se análise descritiva das variáveis estudadas através da frequência absoluta e relativa, utilizando o teste qui-quadrado para avaliação da associação do sexo com o transtorno de ansiedade. Considerou-se um intervalo de confiança de $95 \%$ para todas as análises.

\section{RESULTADOS E DISCUSSÕES}

Dos 131 estudantes analisados, 58\% eram do sexo feminino e $42 \%$ eram do sexo masculino e constatou-se que $32,1 \%$ dos alunos participantes possuíam algum Transtorno de Ansiedade (TAs). Esse valor aumentado nesta população pode ser comparado a um estudo realizado com indivíduos com idade entre 18 e 35 anos no estado de São Paulo, no qual constatou-se a presença de TAs em 26,4\% desses (COSTA et al., 2019).

Tabela 1 - Distribuição do sexo, estado nutricional e transtornos de ansiedade em 131 adolescentes escolares. Campina Grande - PB, 2016.

\begin{tabular}{lll}
\hline \multicolumn{1}{c}{ VARIÁVEIS } & $\mathbf{n}$ & \% \\
\hline Sexo & & \\
Masculino & 55 & 42,0 \\
Feminino & 76 & 58,0 \\
Transtornos de Ansiedade (TAs) & 42 & 32,1 \\
Presença Ausência & 89 & 67,9 \\
\hline
\end{tabular}

Fonte: Dados da Pesquisa, 2016.

Estes valores aumentados com adolescentes nesta faixa etária também podem estar relacionados com fatores relativos à conclusão do ensino médio, realização de exames vestibulares, pressão psicológica familiar, tomada de decisão a respeito de escolhas profissionais entre outros (GROLLI; WAGNER; DALBOSCO, 2017). 
Além disso, as estudantes do sexo feminino apresentaram maior prevalência de Transtornos de Ansiedade (40,8\%) que os estudantes do sexo masculino (23,6\%). Tal resultado pode ser comparado com um estudo realizado com adolescentes escolares entre 16 e 19 anos, matriculados em escolas públicas do interior do Estado do Rio Grande do Sul, no qual constatou-se níveis de ansiedade mais elevados em adolescentes do sexo feminino. Isso associa-se a diversos fatores pertinentes à adolescência, variabilidade hormonal e outras situações inerentes ao sexo feminino (GROLLI; WAGNER; DALBOSCO, 2017).

Tabela 2 - Distribuição dos Transtornos de Ansiedade em 131 adolescentes escolares de acordo com o sexo. Campina Grande - PB, 2016.

\begin{tabular}{lcccc}
\hline & \multicolumn{2}{c}{ SEXO } & & \\
& Masculino & Feminino & RP (IC 95\%) & $\boldsymbol{p}$ \\
\hline VARIÁVEIS & $\mathbf{n ~ ( \% )}$ & $\mathbf{n ~ ( \% )}$ & & \\
Transtornos de & & & & \\
Ansiedade (TA) & & & & \\
Presença & $11(20,0)$ & $31(40,8)$ & $0,363(0,162-0,811)$ & 0,012 \\
Ausência & $44(80,0)$ & $45(59,2)$ & & \\
\hline
\end{tabular}

Fonte: Dados da pesquisa, 2016.

Constatou-se, então, que houve prevalência significativa de adolescentes com algum tipo de Transtorno de Ansiedade $(p<0,05)$. Além disso, foi observada a maior taxa destes números em adolescentes do sexo feminino, resultado que reflete e é somado com diversos outros estudos que demonstram esta associação com o gênero.

\section{CONCLUSÃO}

Apesar dos TAs terem impacto significativo no estilo de vida dos adolescentes, esse tema é pouco explorado pela literatura Kinrys e Wygant (2005) em consonância com este estudo, apontam que, além de haver uma maior 
prevalência de TAs em pessoas do sexo feminino, as apresentações clínicas e as características individuais de cada transtorno pode variar de acordo com o sexo.

A etiologia da doença ainda é controversa, porém fatores genéticos e ambientais têm sua contribuição no que diz respeito ao desenvolvimento desses transtornos, além da intensa atividade hormonal e dos ciclos femininos que podem determinar o desenvolvimento dos TAs em mulheres (BATISTA; DE OLIVEIRA, 2016, DA SILVA FILHO; SILVA, 2017).

Os hormônios femininos têm sua contribuição no que concerne a frequência, gravidade e curso dos TAs. Ainda, os ciclos menstruais, a resposta às situações de medo e perigo desencadeiam maiores respostas emocionais quando comparado ao sexo oposto. Evidências científicas sugerem que isso pode acontecer pelo fato do córtex anterior do giro do cíngulo ser anatomicamente maior e mais ativa nas mulheres, e isso causa respostas emocionais e racionais mais intensas no sexo feminino, elevando as tensões e os níveis de ansiedade (KINRYS, 2005).

Por outro lado, esses resultados podem ser explicados por questões apontam a relevância do papel da mulher e os aspectos emocionais envolvidos nas relações familiares e sociais. Diversas mudanças ocorridas com o passa dos anos, decorrentes dos movimentos sociais, alteraram a posição da mulher na sociedade, especialmente a adolescente, tornando-as tão permissíveis quanto os homens. Assim, ambos contribuem para a imposição de novos modelos de relação social e familiar contemporâneos, reduzindo as perspectivas ocasionam desgaste emocional e acarreta em acúmulo de tensões que convergem para o desenvolvimento dos TAs e sua prevalência nessa população. (BORSA; NUNES, 2017).

Outros fatores de origem orgânica e condições clínicas não psiquiátricas podem desencadear um TAs, devendo ser realizadas avaliações clínicas e laboratoriais para melhor compreensão do caso. Sinais e sintomas atípicos apresentados pelo adolescente, num quadro de ansiedade, merecem atenção especial para investigação clínica, a exemplo de: vertigem, ausência de consciência, alteração do padrão de marcha, ausência de controle dos esfíncteres, cefaleia, amnésia e disfunção fonética (DA SILVA FILHO; SILVA, 2017).

Em outra análise dos dados deste estudo, constatou-se que $47,4 \%$ das adolescentes apresentam TP, mostrando significância para o sexo feminino. Isso 
reforça a hipótese de Kessler (2005) que, utilizando dados do National Comorbidity Survey - NCS nos Estados Unidos, relataram que o transtorno do pânico foi mais prevalente entre mulheres entre 15 e 24 anos. Diversos estudos indicam que as mudanças hormonais inerentes ao sexo feminino têm impacto considerável na etiologia clínica do TP em mulheres.

Sabe-se que o Brasil apresenta-se como o país com maior taxa de pessoas com TAs, atingindo 9,3\% dos brasileiros (ANDRADE et al., 2019). Portanto, pode-se concluir que se faz necessária a investigação da ocorrência de Transtornos de Ansiedade entre os adolescentes, através de estudos com delineamento longitudinal, com o objetivo de traçar estratégias de promoção e prevenção de saúde mental com estes indivíduos no contexto familiar e escolar, promovendo a redução destes índices. Ainda, faz-se necessário estudos que estabeleçam causalidade entre a presença de TAs e o sexo feminino, além de alternativas que corroborem para a diminuição destes índices na população geral.

\section{REFERÊNCIAS BIBLIOGRÁFICAS}

ALMEIDA, R. S. O uso dos grupos terapêuticos no tratamento dos transtornos de ansiedade sob o olhar da terapia cognitivo-comportamental. Cadernos de Graduação: Ciências Biológicas e da Saúde, v. 3, n. 3, p. 101-118, 2016.

ANDRADE, J. V. et al. Ansiedade, um dos problemas do século XXI. Revista de Saúde ReAGES, v. 2, n. 4, p. 34-39, 2019.

BATISTA, Jefferson Isaac; DE OLIVEIRA, Alessandro. Efeitos psicofisiológicos do exercício físico em pacientes com transtornos de ansiedade e depressão. Revista Corpoconsciência, v. 19, n. 3, p. 1-10, 2016.

BORSA, Juliane Callegaro; NUNES, Maria Lucia Tiellet. Aspectos psicossociais da parentalidade: o papel de homens e mulheres na família nuclear. Psicologia Argumento, v. 29, n. 64, 2017.

COSTA, C. O. et al. Prevalência de ansiedade e fatores associados em adultos. Jornal Brasileiro de Psiquiatria, v. 68, n. 2, p. 92-100, 2019.

DA SILVA FILHO, Orli Carvalho; DA SILVA, Mariana Pereira. Transtornos de ansiedade em adolescentes: considerações para a pediatria e hebiatria. Adolescencia e Saude, v. 10, n. 3, p. 31-41, 2013.

DA SILVA, Guidélia Aparecida; LANGE, Elaine Soares Neves. Imagem corporal: a percepção do conceito em indivíduos obesos do sexo feminino. Psicologia argumento, v. 28, n. 60, 2017. 
GROLLI, V.; WAGNER, M. F.; DALBOSCO, S. N. P. Sintomas depressivos e de ansiedade em adolescentes do ensino médio. Revista de Psicologia da IMED, v. 9, n. 1, p. 87-103, 2017.

GUIMARÃES, A. M. V. et al. Transtorno de ansiedade: um estudo de prevalência sobre as fobias específicas e a importância da ajuda psicológica. Cadernos de Graduação: Ciências Biológicas e da Saúde, v. 3, n. 1, p. 115-128, 2015.

KINRYS, Gustavo; WYGANT, Lisa E. Transtornos de ansiedade em mulheres: gênero influência o tratamento? Revista Brasileira de Psiquiatria, 2005.

LOPES, C. S. et al. ERICA: prevalência de transtornos mentais comuns em adolescentes brasileiros. Revista de Saúde Pública, v. 50, supl. 1, 14s, 2016.

MANOEL, Cristina Landgraf Lee. Ansiedade competitiva entre sexos: uma analise de suas dimensões e seus antecedentes. Revista Paulista de Educação Física, v. 8, n. 2, p. 36-53, 2017.

MÉA, C. P. D.; BIFFE, E. M.; FERREIRA, V. R. T. Padrão de uso de internet por adolescentes e sua relação com sintomas depressivos e de ansiedade. Psicologia Revista, v. 25, n. 2, p. 243264, 2016.

SILVA, C. B. Crianças e adolescentes com fobia específica: intervenções em terapia cognitivocomportamental. Revista Humanae, v. 10, n. 2, p. 1-16, 2016.

SOUZA, Maria Augusta Mansur de. Terapia cognitivo-comportamental em grupo para préadolescentes com transtornos de ansiedade: desenvolvimento das sessões e avaliação de resposta. 2011. 